\title{
$\mathrm{EFD} / \mathrm{JFL}$
}

\section{Das Türken - Und Türkeibild in Helmuth Graf Von Moltkes Briefen (1835-1839)}

Helmuth Graf Von Moltke'nin Mektuplarında (1835-1839) Türk ve Türkiye İmgesi

Mutlu ER*

\section{Zusammenfassung}

Die bilateralen Beziehungen zwischen dem Osmanischen und Preußischen Reich gehen bis auf die Mitte des 19.Jh. zurück. Zu dieser Zeit stiegen im Westen hochkonjunkturelle Imperien auf und das Deutsche Reich versuchte in dieser Konjunktur einen Platz zu halten, indem es mit dem Osmanischen Reich auf militärischer Ebene Kontakt aufnahm. Zu diesem Zweck wurde in den 1830'er Jahren eine Gruppe von deutschen Offizieren für die Militärreform im Osmanischen Reich beauftragt. Unter diesen Offizieren befand sich auch Helmuth Graf von Moltke, dessen Reise durch das Osmanische Reich den dienstlichen Zweck hatte, den Stand der osmanischen Truppen festzustellen, um die entsprechenden Erfordernisse für eine militärische Reorganisation per Bericht zu verfassen. Moltke schrieb seine Eindrücke über die Menschen und das Leben in Briefen nieder, die später in einem Buch zusammengebracht und veröffentlicht wurden. Neben dem Türkenbild kann auch das Türkeibild aus seinen Briefen entnommen werden. Des Weiteren bezeichnet er den anatolischen Bereich in seinen Briefen als Kleinasien. Von Helmuth Graf von Moltkes Erfahrungen profitierte der deutsche Reformausschuss, der in den 1880'er Jahren den Arbeiten einen Aufschwung zu geben erwünschte.

Schlüsselwörter: Helmuth Graf von Moltke, Reise, Briefe, Türkenbild, Türkeibild, Bericht, Militärreform, historische Begebenheiten

$\ddot{O} z$

Tarihte Osmanlı İmparatorluğu ve Prusya Krallığı (Almanya) arasındaki resmi ilişkiler 19.yy’ın ortalarına denk gelmektedir. Bu dönemde Batı'da sanayi imparatorlukları yükselmeye başlamış ve Alman İmparatorluğu da bu yeni düzende yerini almak üzere Osmanlı İmparatorluğu ile askeri alanda ilişkiler kurmuş ve bu alandaki reformları gerçekleştirmek üzere Alman Islah

* Öğretim Görevlisi Dr., Hacettepe Üniversitesi, Alman Dili ve Edebiyatı Bölümü, mutluer@hacettepe.edu.tr 
Heyeti‘nin ilk subaylarını 1830'lu yıllarda Osmanlı İmparatorluğu’na göndermiştir. Subaylardan oluşan bu heyette genç bir subay olan Helmuth Graf von Moltke Osmanlı Ordusu'nun durumunu yerinde görmek ve tespit etmek için bir heyet eşliğinde yola çıkmış ve askeri alandaki teknik raporların yanı sıra Türkler ve Osmanlı İmparatorluğunun sosyal düzeni hakkındaki tüm izlenimlerine yer verdiği mektuplar yazmıştır. Moltke'nin dönüşünden sonra bu mektuplar bir kitapta toplanmış ve yayınlanmıştır. Türk İmgesinin yanısıra bir de Türkiye İmgesi hakkında bilgiler içeren bu mektuplarda İmparatorluğun Anadolu topraklarını Küçük Asya diye adlandırmıştır. Alman Islah Heyetinin çalışmalarına hız verdiği 1880'li yıllarda Moltke'nin bu tecrübeleri daha sonra atılacak olan adımlarda yol gösterici olmuştur.

Anahtar Sözcükler: Helmuth Graf von Moltke, Seyahat, Mektuplar, Türk İmgesi, Türkiye İmgesi, Rapor, Islah Hareketi, Tarihsel Olaylar

\section{Einleitung}

Helmuth (Karl Bernhard) Graf von Moltke, der von einer mecklenburgischen Familie stammt ist am 26.10.1800 in Mecklenburg geboren. Er wuchs im dänischen Holstein auf und besuchte von 1811 bis 1817 die Kadettenakademie in Kopenhagen. Im Jahre 1818 wurde er Page am Königshof, von 1819 bis 1822 war er Leutnant in dänischen Diensten und trat 1823 in die preußische Armee ein. 1832 wurde er in den Großen Generalstab versetzt (Jessen, 2010). 1835 erhielt er eine Genehmigung zu einer Bildungsreise in den Südosten Europas. Auf Wunsch des damaligen Sultans wurde Moltke mit Fincke, Fischer und Mühlbach (Jessen, 2010, S. 80) von 1836 bis 1839 als Instrukteur der Osmanischen Truppen abkommandiert. Obwohl er während seiner Dienstzeit Istanbul verlassen wollte, wurde dieser Wunsch abgelehnt, da der Sultan seine militärische Mission weiterzuführen wünschte. Bei seinem Aufenthalt arbeitete er an topographischen Aufnahmen und unternahm Reisen in Kleinasien, die auf der nachkommenden Abbildung angezeigt werden (Kahlmeyer, 2002, S. 12):

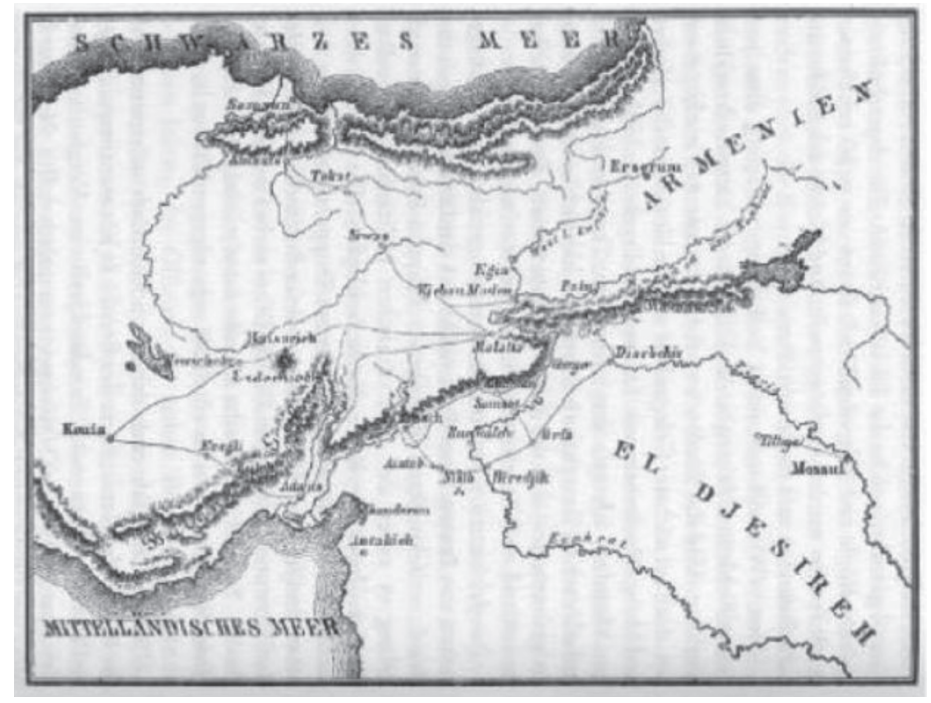


Bei seinem fünf jährigen Aufenthalt schrieb Moltke Briefe, die er nach seiner Heimkehr weitergeleitet hat (Kahlmeyer, 2002, S. 13). Dadurch entstand Moltkes Werk aus 65 Briefen, in denen sein Türken und Türkeibild erscheinen. Im Folgenden wird versucht in den Kapiteln aus Graf Moltkes Reiseberichten das Türken- und Türkeibild herauszuschreiben. Da es zu Anfang des 19 Jahrhunderts Beobachtungen von einem deutschen General darstellt und sich die Deutschen und Türken zum ersten Mal in der Geschichte auf einer friedlichen Ebene annähern ist das Werk für Beobachtungen bemerkenswert. Schon nach über 170 Jahren entnehmen wir seinem Türkenbild, dass schon damals das Image der Türken in den Gedanken der Europäer mittelalterlich und zurückgeblieben oder als nicht reichlich zivilisiert verstanden worden ist.

Unter dem Titel "Halbmond" ist eigentlich der Orient zu verstehen, da zu diesem Zeitpunkt ein Teil der arabischen Länder unter der Machtführung des Osmanischen Reiches stand. Es stehen auch Vergleichungen über das Leben der Türken mit Arabern, Griechen, Armeniern usw. Obwohl die Briefe zu den Zeiten des Osmanischen Reiches verfasst worden sind, spricht Moltke nicht vom Osmanischen Reich, sondern von der Türkei. Diesbezüglich kann man die Stereotypen als die der Türkei und Türken verstehen, wie in der Überschrift dieses Artikels erwähnt wurde. Die Gedanken und Bezeichnungen Moltkes über die Kultur der Türken werden expliziert.

\section{Beobachtungen und Wertungen in Moltkes Briefen}

Seinen ersten Brief schrieb Moltke am 25.Oktober 1835 in Bukarest mit dem Titel "Besuch beim Pascha von Neu-Orsowa. Reise durch die Wallachei". Seine ersten Eindrücke bei der Begegnung mit den Menschen und der türkischen Provinz sind sehr negativ. Moltke bezweifelt vor allem, dass nach einer Besetzung einer Stadt keine Umbauungen oder jegliche Restaurationen an Gebäuden durchgeführt worden sind:

In der Stadt überraschte und die Unreinlichkeit der engen Straßen. Die Anzüge der Männer waren roth, gelb, blau, kurz von den schreiendsten Farben, aber alle zerlumpt. Die Frauen schlichen tief verhüllt wie Gespenster umher. Alle Wohnungen trugen Spuren des Zerfalls, und an der Festung ist, glaub' ich, seit der Besitznahme kein Ziegel ausgebessert (Moltke, 1882, S. 2).

Aus diesem Zitat kann man entnehmen, dass die Spuren der Auflösung des Osmanischen Reiches im 19.Jh. an den unreinen Straßen und den zwar farbenfrohen jedoch abgetragenen Bekleidungen der Menschen zu sehen ist. Die türkische Frau hinterlässt bei Moltke die Empfindung, sie lebe abgedrängt vom gesellschaftlichen Leben. Die Bekleidung der Türken wird in Moltkes Briefen ("Reise nach Samsun - Die Häfen des Schwarzen Meeres", 1838) besonders hervorgehoben, da sie zwar einerseits auf den sozialen Status deutet, andererseits aber, wenn veraltete Bekleidungen nur vom Volk getragen werden, sie die wirtschaftliche Lage der Menschen widerspiegeln: 
Der Reisenden sind stets viele, aber fast nur Verdeckt=Passagiere; der Türke führt sein Bett, sein dürftiges Mahl und seine Pfeife mit sich, wickelt sich Nachts in seine Pelze und Teppiche, und verlässt fast nicht den Platz, auf welchen er sich bei der Abfahrt hinsetzt (Moltke, 1882, S. 199).

In seinem Brief "Die orientalische Nacht", die er 1838 schrieb, wird sogar berichtet, dass sich das normale Volk aus Ehrfurcht und Bekenntnis seines eigenen gesellschaftlichen Standes schlecht bekleidet:

Die Türken steigen in demselben Anzug zu Pferde, in welchem sie schlafen, und brauchen weder Sprungriemen noch Sporen anzulegen. Niemand braucht ein anderes Kleid anzuziehen, weil er zu einem vornehmen Manne geht[...](Moltke, 1882, S. 306).

Aus dem Brief vom 29.November 1835 mit der Überschrift "Walachische Schlitten Gjurgewo - Rustschiik - Reise mit dem Tataren - Schumla - Türkische Bäder - Der Balkan - Adrianopel - Ankunft in Konstantinopel”, die Moltke später in Istanbul verfasste, werden folgende Bemerkungen über den Zustand der Bauwesen gemacht:

Endlich erreichten wir das Hann oder den türkischen Gasthof[... JIrgendein reicher Pascha erbaut ein solches Hotel als eine Art fromme Stiftung, aber Niemand denkt daran, es zu möblieren, oder nur in baulichem Stande zu erhalten [...] Mir fiel es besonders auf, daß in einer Stadt, die einen so rauhen Winter hat, wie hier an den Ufern der Donau, nicht einmal Fensterscheiben zu finden waren. Die Fenster waren entweder ganz offen, oder höchstens mit Papier verklebt (Moltke, 1882, S. 12).

Aus diesen Sätzen empfindet man, in welch einer Gleichmütigkeit die Türken leben. Zwar begegnet Moltke einige Neubauten, die nach der Eroberung erstellt worden sind, aber im Vergleich zu den europäischen Gebäuden in einem ungepflegten Zustand und unvollkommen sind. Eine ähnliche Beobachtung verfasste Moltke im Brief "Reise nach Brussa” im Jahre 1836:

Wie bei allen türkischen Städten, so auch hier, verschwindet das prächtige Bild, sobald man in die Stadt hineintritt. Der kleinste deutsche Marktflecken übertrifft Konstantinopel, Adrianopel und Brussa an Zierlichkeit der Wohnungen, und noch mehr an Bequemlichkeit. Großartig sind nur die Moscheen und die Hanns oder Karavanserais, die Fontainen und öffentlichen Bäder (Moltke, 1882, S. 6).

Im Vergleich zu den kleinen feudalen deutschen Städten sind Istanbul, Edirne und Bursa (die letzteren zwei waren auch sehr bedeutsame Städte des Osmanischen Reiches) unausgewogen und unansehnlich. Nur die Bauten, die im Zitat erwähnt worden sind 
(Moscheen, Gasthöfe, Karawansereien, Springbrunnen und Bäder), widerspiegeln die Herrschaft des Osmanischen Reiches. Weder der Lebensstandard der Bourgeoise noch die des Volkes in den Städten scheint in Moltkes Bemerkungen kein Ansehen zu haben. Anders beschreibt Moltke die Lage in seinem Brief "Reise nach Iconium" (1838) über Konya. Hier wird ein sehr negatives Bild über die Türken geprägt. Des Weiteren wird auch an die vernichtenden Umbauungen der Kulturerben eine scharfe Kritik ausgeübt. Weder die Herrschaften der Christen noch der Muslime hätten keine Rücksicht auf andere Kulturen gehabt:

Die türkischen Städte haben überhaupt das Ansehen der Verödung, aber keine mehr als Konieh; es ist weniger verfallen durch die Zeit, als zerstört durch Menschenhände. Ein Jahrhundert hat hier immer seine Denkmäler erbaut aus den Trümmern der vorhergehenden; in der christlich=römischen Zeit riß man die Tempel ein, um Kirchen zu erbauen; die Moslems verwandelten die Kirchen in Moscheen und die Moscheen liegen heut in Trümmer (Moltke, 1882, S. 318).

Bei einem diplomatischen Treffen kann man die Äußerungen Moltkes (aus dem Brief: Der Frühling am Bosporus - Türkisches diplomatisches Mittagessen-Pera, den 20. Mai 1836) zu der Gastaufnahme, Küche und Essgewohnheiten der Türken folgendes entnehmen:

Da diese Feier echt türkisch ist, so gab man uns auch ein echt türkisches Diner, natürlich ohne Messer und Gabeln und ohne Wein. Den Anfang der Schüsseln machte ein gebratenes Lamm, inwendig mit Reis und Rosinen gefüllt. Jeder riß sich ein Stück ab und langte mit den Fingern hinein; dann folgte Helwa, eine süße Mehlspeise aus Honig, dann wieder Braten und wieder ein süßes Gericht, bald warm, bald kalt, bald sauer, bald süß. Jede einzelne Schüssel war vortreffich, die ganze Kombination aber für einen europäischen Magen schwer begreiflich, und das Alles ohne Wein (Moltke, 1882, S. 61-62).

Man kann aus diesem Zitat ableiten, dass Moltke eine relativ sehr ungewohnte Situation beim Essen beschreibt, da die Türken beim Essen kein Besteck benutzen. Außer der unfeinen Einstellung der Türken beim Essen, versteckt sich überdies eine andere kritische Aussage über die orthodoxe Einstellung der Türken beim Empfang eines nichtmuslimischen Gastes, da, wie es sonst für einen Europäer üblich ist, trotz einer übertriebenen Großzügigkeit des Mahls kein Wein (im Zitat zwei Mal betont) eingeschenkt worden ist. Diesbezüglich wird auf eine andere Lage hingewiesen. Diese für Moltke sehr unangenehme Lage wurde im Brief "Rückkehr nach Konstantinopel" geschildert, als Moltke 1839 in Samsun in ein österreichisches Dampfboot, das sie aus der asiatischen Barbarei in die europäische Verfeinerung (Moltke, 1882, S. 403) fahren sollte, einstieg:

In unserer zerlumpten türkischen Kleidung, mager und abgezehrt, mit langen Barten und türkischem Gefolge, wollte man uns erst gar nicht in 
die erste Kabine lassen, bis wir den Kapitän auf Französisch anredeten. Es ist nicht zu beschreiben, wie behaglich uns alles vorkam; da gab es Stühle, Tische und Spiegel, Bücher, Messer und Gabeln, kurz, lauter Bequemlichkeiten und Genüsse, deren Gebrauch wir fast verlernt hatten (Moltke, 1882, S. 403).

Aus dem ersten Satz dieses Zitates kann man ein Stereotyp entnehmen: veraltete und schmutzige Kleider kann nur ein Türke anziehen. Dass Moltkes Aussehen einem Türken wahrhaftig nah gewesen ist verhinderte ihn zuerst in den Dampfer einzusteigen. Als die Barbarei bezeichnet Moltke eine Unbildung der Türken. Der Gebrauch von den oben erwähnten Gegenständen stellt eine Erfordernis der Modernisierung dar und zeigt deutlich die tiefe kulturelle Kluft zwischen beiden Kulturen.

Neben diesen negativen Bewertungen von Moltke gibt es in den Briefen auch positive Auffassungen über die türkische Gastfreundlichkeit:

[...] überschritten bei fortwährendem Regenwetter den Balkan und ruhen uns jetzt in Varna aus, wo uns der Pascha aufs Zuvorkommendste aufgenommen, und wo man für uns so gut gesorgt hat, wie es die Umstände erlauben (Moltke, 1882, S. 157).

Das oben angeführte Zitat aus dem Brief "Reise durch Rumelien, Bulgarien und die Dobrudscha - Der Trajanswall" von 1837 ist ein Beispiel für eine relativ feine Umgangsform mit einem fremden Gast. In einem anderen Brief "Ritt durch das Gebirge vom Tigris an den Euphrat" von 1838 beschreibt Moltke seinen Besuch bei einem Gouverneur in seinen Einzelheiten wie folgt:

Wenn in der Türkei ein Mann von einiger Bedeutung ankommt, so ist es unerlässlich, dass einige der vornehmsten Einwohner ihm schon vor der Stadt entgegengehen, man hilft ihm vom Pferde, stïtzt ihn, wenn er die Treppe hinaufsteigt, zieht ihm die Stiefel aus und legt ihn auf das Kissen rechts vom Kamin. Der Müsselim, oder wer der Herr des Hauses sein mag, räumt sogleich das Zimmer; er läßt sich nur auf ausdrücklicher Einladung und in der Nähe der Thür auf dem bloßen Fußboden nieder, und wenn man ihm gestattet, von seinem eigenen Kaffee zu trinken, so empfängt er ihn mit einer tiefen Verbeugung und dem Gruße mit der Hand an die Erde. »Das Haus ist Deins« ist [...] (Moltke, 1882, S. 292293).

Die detaillierte Darstellung der Aufnahmebereitschaft der Türken während eines offiziellen Besuches kann insofern als sehr positiv verstanden werden, da diese Tradition auch wortwörtlich durchgeführt wurde und seit Moltkes erste Begegnung mit den Türken eine Bewertung ist, die frei von Ironie ist. Was auf der einen Seite Moltke sehr positiv beeindruckt hat, ist die Beschenkung eines Gastes, wurde jedoch von ihm nicht gut verstanden: 
[...], so lange man bleibt, nicht bloß die übliche Redensart, und ein solcher Gast muss zum Abschiede noch obendrein reichlich beschenkt werden. [...] Mir führte der Müsselim ein junges Pferd, [...] er war sehr betreten, dass ich mich weigerte, sein Geschenk anzunehmen, und betheuerte, daß in der ganzen Stadt kein edleres Thier zu haben sei[...] (Moltke, 1882, S. 292-293).

Die im obigen Zitat beschriebene Situation bezeugt, dass sich beide Kulturen sehr fremd sind. Weder Moltke noch der Gouverneur sind imstande zu begreifen, wie unterschiedlich eine Beschenkung zu verstehen ist. Die europäische Einstellung Moltkes zeigt sich im Brief "Reise nach Iconium" (1838) als eine Intoleranz gegen eine zeitverschwenderische und nachlässige Form. Die formal strengen Regeln der Bürokratie stellen der Reform ein großes Hindernis in den Weg:

Nun kenne ich aber meine Türken gut genug, um zu wissen, daß hier durch Warten oder Nachgeben nichts zu gewinnen war; ich erklärte daher dem versammelten Schwarm von Kawassen und Agas zuversichtlich und laut, daß ich unverzüglich zum Müsselim geführt zu werden beabsichtige, daß ich nicht gewohnt sei, mich im Hof empfangen zu lassen (Moltke, 1882, S. 315).

Neben den verwüsteten Städten, Mahlzeiten ohne Wein und den ausgenutzten Kleidern, wo der Genuss am Leben fehlt, bemerkt Moltke im Brief "Der Ramadan" im Jahre 1838, dass die Türken eine starke Abhängigkeit am Tabakrauchen haben und nicht nur bei Gelegenheiten, sondern auch bei wichtigen Besprechungen die Pfeife dabei haben:

Da es unseren Freunden und Genossen, den Türken, unmöglich ist, $z u$ arbeiten ohne zu rauchen, so geschehen jetzt alle Geschäfte des Nachts; die Kanzlei ist versammelt, Briefe werden gelesen und spediert, Meldungen angenommen, Geschäfte besprochen (Moltke, 1882, S. $331)$.

Der übertriebene Konsum an Tabak zeigt sich im Brief "Stilleben von Bujukdere", der im Jahre 1837 verfasst wurde. Man kann aus Moltkes Brief entnehmen, dass die Türken- Männer und Frauen sind hier gemeint- vom kriegerischen und vitalen Volk in der Auflösungsperiode des Osmanischen Reiches zu einem melancholischen und faulen Genussmenschen geworden sind:

Man begreift nicht, wie die Türken haben leben können, ehe die große Erfindung der Pfeife gemacht wurde. Wirklich waren die Gefährten Osmans, Bajasids und Mehmeds ein turbulentes Volk, das beständig im Sattel lag und Länder und Städte eroberte. Seit Suleimans Zeiten haben sie ihre Nachbarn auch wohl noch manchmal heimgesucht, sind aber doch ein wesentlich sitzendes und heute ein wesentlich rauchendes 
Volk geworden, denn selbst die Frauen »trinken« den Tschibuk (Moltke, 1882, S. 146).

Was in Betracht auf die Trägheit der Türken als ein sitzender Mensch in Moltkes Briefen verfasst worden ist, so zeigt sich auch die türkische Beschaffenheit im Gebrauch der Verkehrsmittel im Brief “Reise nach Orfa” (1839), die für einen Europäer nicht leicht aufzuklären scheint:

Die Türken begreifen überhaupt nicht, wie Jemand, der ein Pferd oder einen Esel hat, zu Fuß gehen kann; sie stehen still und sehen nach: “Iürir”-erwandert-sagen sie erstaunt (Moltke, 1882, S. 343).

Trotz der negativ geschilderten Lage der Menschen fällt Moltke die Tierliebe der Türken ins Auge. Es sind vor allem Stiftungen, die vom Sultan gegründet worden sind, um die Barmherzigkeit anzuregen und hervorzuheben. Im folgenden Zitat aus dem Brief “Amasia” vom Jahre 1838 kann die Pflege der Straßentiere abgelesen werden:

Der Wohltätigkeitssinn der Türken dehnt sich bis auf die Thiere aus. In Skutari findest Du ein Katzen=Hospital, und in dem Vorhof der Moschee Bajasids giebt es eine Versorgungsanstalt für Tauben (Moltke, 1882, S. 102).

Die Tiere, die nicht von einer Stiftung betreut werden, finden entweder Fürsorge vom Volk oder müssen sich von den Resten der Menschen begnügen, da der Müll auf den Straßen nicht gereinigt wird. Die Ironie lässt sich im selben Brief, “Amasia”, wie folgt ablesen:

In den Häusern findet man niemals Hunde, aber in den Straßen leben viele Tausende dieser herrenlosen Thiere von den Spenden der Bäcker, der Fleischer und freilich auch von ihrer Arbeit, denn die Hunde haben hier fast ganz allein das Geschäft der Straßenreinigungs=Komissaire übernommen (Moltke, 1882, S. 102).

Neben dem durch detaillierte Beobachtungen in den Briefen dargestellte Türkenbild, in dem die Menschen mehr in einer trüben Situation wahrgenommen werden können, zeigen sich auch positive Darstellungen im Brief “Amasia” (1838):

Der Anblick von der Zitadelle herab ist prachtvoll; es war eben Beiram, der größte Feiertag der Türken. Ueberall war Leben, und sämtliche Frauen, in ihren grellen bunten Gewändern, kamen aus den Bädern. Von der Zitadelle wurde mit Böllern geschossen, die in den Thälern prächtig widerhallte [...] (Moltke, 1882, S. 202).

Es kann aus diesen Sätzen, in dem die Türken bunt bekleidet, gepflegt und mit Freude auf den Straßen eine heitere Lage darstellen, entnommen werden, mit welch einer Begeisterung Moltke die Menschen beschrieben hat. Bei einem anderen Fest, 
im Brief "Der Ramadan" von 1838, stellt Moltke fest, wie durch Beschenkungen und Aufrechterhalten der Traditionen der Markt einen Gewinn ziehen kann:

In acht Tagen haben wir nun den Beyram, was bei uns etwa das Osterfest ist, ein Fest der Freude, der Gratulationen und der Geschenke; Jedermann giebt und empfängt an diesem Tage, wie überhaupt »Almalyvermaly! « der Wahlspruch der Türken ist: »Nehmen und geben« oder "Leben und leben lassen", dies ist in der That eine einfache und angenehme Staatswirthschaftslehre; alle Klassen der Gesellschaft profitiren davon [...] (Moltke, 1882, S. 332-333).

Moltkes negative Bemerkungen sind teilweise als Stereotypen zu verstehen, aber bei der Feststellung der Türkei- und Türkenbilder kann man bemerken, dass Moltke versuchte die Lage zu analysieren und detailliert anzugeben, um diese auch begründen zu können, da wegen seines Auftrags als Feldmarshall diese Bemerkungen wertvoll gewesen sind. Im letzten Jahr seines Türkeiaufenthalts beschrieb er im Brief "Sultan Mahmud II.", die er 1839 verfasste, aus welchen Gründen das Osmanische Reich zum kranken Mann am Bosporus wurde:

In seinem eigenen Volke fand Sultan Mahmud auch nicht einen erleuchteten Mann, der ihm bei seinen Neuerungen leitend oder helfend zur Seite gestanden hätte; es ist den Europäern fast unmöglich, sich den Standpunkt der Intelligenz im Orient so niedrig zu denken, wie er wirklich ist. Ein Türke, welcher lesen und schreiben kann, heißt »Hafiz«, ein Gelehrter; die Kenntni $\beta$ des ersten und letzten Verses aus dem Koran vollendet seine Bildung und die vier Spezies sind den wenigsten geläufig. [...]Niemand spricht irgendeine europäische Sprache, außer etwa die Renegaten, und viele Türken in hohen Ämtern müssen sich die Briefe, welche sie in ihrer eigenen Sprache erhalten, vorlesen lassen [...] (Moltke, 1882, S. 411-412).

Eine sehr bedeutende Rolle beim Rückgang des Osmanischen Reiches spielte dem obigen Zitat nach das Fehlen aufgeklärter Menschen an der rechten Seite des Sultans. Moltke stellt die Verstandeskraft der Türken im Brief "Sultan Mahmud II." (1839) in Frage:

Es blieb demnach nichts weiter übrig, sich Rath bei den Fremden zu holen; aber in der Türkei wird die beste Gabe verdächtig, sobald sie aus der Hand eines Christen kommt. Peter der Große hatte 500 Offiziere, Ingenieure, Artilleristen, Wundärzte und Künstler für seinen Dienst persönlich angeworben; sie theilten seine Mühe und ernteten die Früchte derselben. In Russland konnten die Fremden gehasst sein, in der Türkei sind sie verachtet. Ein Türke räumt unbedenklich ein, dass die Europäer seiner Nation an Wissenschaft, Kunstfertigkeit, Reichthum, Kühnheit und Kraft überlegen seien, ohne daß ihm entfernt in den Sinn käme, daß 
um Deswillen ein Franke sich einem Moslim gleichstellen dürfte[...]

(Moltke, 1882, S. 412).

Moltkes Feststellung nach fehlt im Osmanischen Reich ein Gelehrtentum, das mit Rat an der Seite des Sultans steht. Aus diesem Grund weist er auf Berater, die aus Europa eingeladen werden sollten, um dieses Bedürfnis decken zu können. Hier jedoch ist Moltke sehr skeptisch, da man sich von einem Christen, der damals als ein Ungläubiger angesehen wurde, nicht bereiten ließ. Bei der oberen Schicht, sowie den Untertanen des Sultans sind jegliche westliche Neuerungen unerwünscht (Jessen, 2010:80). Als Beispiel wird Peter der Große angegeben, um ein Kontrastieren zu ermöglichen, da vor allem das Bildungswesen im Osmanischen Reich nicht auf das Wissenschaftliche, sondern auf die orthodoxe Ausbildung angewiesen ist. Mit dieser negativen Gegenüberstellung wollte Moltke hervorheben, wie groß die Lücke zwischen dem Orient und dem Okzident besteht. Andererseits kann man aus diesem Zitat zu dem Gedanken kommen, dass die geplante Militärmission zu scheitern scheint. Diese Kluft zwischen zwei Kulturen und die Frage, ob das Osmanische Reich und die Türken ein Teil Europas seien lässt sich aus dem folgenden Zitat des Briefes "Die Pest" ablesen:

Die Türken fühlen, dass sie in Europa nicht zu Hause sind, ihre Prophezeiungen und Ahnungen sagen ihnen, dass das Römische Reich ihnen nicht immer gehören werde, und wer die Mittel dazu hat, lässt seine Asche auf die asiatische Seite des Bosphorus nach Skutari bringen (Moltke, 1882, S. 104).

Die Prophezeiung, von der Moltke in diesem Brief erwähnt hat, ist kein religiöses Vorhersagen, sondern eine Konspiration. Es geht hier darum, dass angeblich die Kasse des Osmanischen Reiches von den Schatzgräbern ausgeplündert und an vielen unterschiedlichen Stellen versteckt sei. Diese Beobachtung zeigt, dass die Türken einen festen Glauben daran gehabt haben, sie seien in den Zeiten des Verfalls des Osmanischen Reiches ihrem Schicksal ausgeliefert. Die Europäer wären demnach nicht imstande gewesen das Osmanische Reich durch Kriegszüge von außen, sondern durch hinterlistige Handlungen von innen zu schwächen und zu erobern. Moltkes Beobachtung nach sehen die Türken den Grund ihres Rückganges nicht an ihrer weltanschaulichen Aufklärung.

\section{Abschließende Betrachtung}

Graf Helmuth von Moltke war in seinen Beobachtungen nicht allein ein Offizier, sondern auch ein Politologe, der die Lage im Osmanischen Reich aus dem Blickwinkel eines Wirtschaftswissenschaftlers betrachten konnte (Önsoy in Ortayl1, 2010, S. 55). Moltke hatte nicht den Status als offizieller Beauftragter unter dem Sultan. Im Jahre 1880 vertrat er als Generalstabchef die Ansicht, dass deutsche Offiziere weiter unter dem Befehl des Kaisers stehen sollten (Ortaylı, 2010, S. 105), denn diese Sache hatte folgende Gründe: 
Die Türkei ist militärisch eine Null! Die Berichte unserer Militärmission lauten geradezu trostlos. Die Armee ist in einer Verfassung, die jeder Beschreibung bespottet. Wenn man früher von der Türkei als vom kranken Mann sprach, so muss man jetzt schon von dem sterbenden Mann sprechen...Unsere Militärmission gleicht einem Arztekollegium, das am Sterbebett eines unheilbar Kranken steht (Neulen in Korn. S. 202).

Eine andere Bemerkung, die das Türkeibild sehr negativ und hoffnungslos darstellt, kann an dieser Stelle von Carsten Niebuhr erwähnt werden, der seine Reiseberichte zwischen den Jahren 1733-1815 im Osmanischen Reich verfasste:

Also Sprache, Religion, politische und militärische Gseze, Vorurtheil, Sitten und Gebräuche trennen die Otmanly von den Europäern, und so müsste es wol durch ein Wunderwerk geschehen sein, wenn selbige die Kriegswissenschaft der Europäer...in so kurzer Zeit gelernt hätten. Gott wird aber wol kein Wunderwerk verrichten, um die Otmanly klug zu machen...(Niebuhr bei Hagen, 2002, S. 317).

Moltkes Pessimismus kann durch dieses Zitat von Carsten Niebuhr unterstrichen werden. Die Wissenschaft, sie sei jedoch hier als eine Ironie zu verstehen, kann dieses orientalische Problem nicht lösen. Eine logische Denkrichtung kann hier von Polaschegg und Korn überliefert werden. Das Osmanische Reich, das einst in der westlichen Literatur mit seiner Wendigkeit, modernen Bewaffnung und effizienten Organisation ungemein schlagkräftig verbreitet war und Respekt abnötigte (Polaschegg, 2005, S. 127), löste sich wegen unsolider Haltung und der unaufgeklärten Bildung auf und Bürokratie und Vetternwirtschaft machten weite Teile des Landes unregierbar (Korn, 2009, S. 72). Infolge der negativen Schlussbemerkungen Moltkes über das Türken bzw. Türkeibildes und dem Prozess der bilateralen Beziehungen, der bis zum 1.Weltkrieg andauerte, können Auf- und Abstiege festgestellt werden. Der deutsche Einfluss im Osmanischen Reich, der Ortaylı nach noch immer in Frage gestellt werden sollte (Ortaylı, 2010, S. 205), hat dazu geführt, dass uns wichtige Berichte überliefert worden sind, in denen die Vorurteile und Stereotypen über Türken verfasst worden sind. 


\section{Bibliographie}

Hagen, G. (2002). "Unter den Tyrannen seiner Araber-Carsten Niebuhr über Konstantinopel, Türken und Osmanisches Reich.”. Bei Carsten Niebuhr (17331825) und seine Zeit. (Herausg. Wiesehöfer, Josef; Conermann, Stephan). (S. 301324). Stuttgart: Franz Steiner Verlag.

Jessen, O. (2010) . Die Moltkes. Biographie einer Familie. München: C.H. Beck Verlag.

Kahlmeyer, A. (2002). Den alten, etwas eingerosteten Krummsäbel alla franca anschleifen. Die Militärmission Helmuth von Moltkes in der Türkei 1835 - 1839. Grinn Verlag.

Kressenstein, F. F. K. (1938). Mit den Türken zum Suezkanal. (Çev. Tahir Balaban). İstanbul: Yeditepe Yayınevi

Moltke, von H. (1882). Briefe über Zustände und Begebenheiten in der Türkei aus den Jahren 1835 bis 1839. Berlin: Königliche Hofbuchhandlung, 4. Auflage.

Ortaylı, İ. (2010). Osmanlı İmparatorluğunda Alman nüfuzu. İstanbul: Timaş Yayınları.

Polaschegg, A. (2005). Der andere Orientalismus. Berlin: De Gruyter Verlag. 